\title{
DEVELOPMENT OF HIGH-PERFORMANCE DETECTOR TECHNOLOGY FOR UV AND IR APPLICATIONS
}

\author{
Ashok K. Sood ${ }^{1}$, John W. Zeller ${ }^{1}$, Parminder Ghuman ${ }^{2}$, Sachidananda Babu', and Nibir K. Dhar ${ }^{3}$ \\ ${ }^{1}$ Magnolia Optical Technologies Inc., 52-B Cummings Park, Woburn, MA 01801 \\ ${ }^{2}$ NASA Earth Science Technology Office, Greenbelt, MD 20771 \\ ${ }^{3}$ U.S. Army Night Vision \& Electronic Sensors Directorate, Fort Belvoir, VA 22060
}

\begin{abstract}
Sensing and imaging for ultraviolet (UV) and infrared (IR) bands has many applications for NASA, defense, and commercial systems. Recent work has involved developing UV avalanche photodiode (UVAPD) arrays with high gain for high resolution imaging. Various GaN/AlGaN $p$-i-n UV-APDs have been fabricated from epitaxial structures grown by metalorganic chemical vapor deposition (MOCVD) on $\mathrm{GaN}$ substrates with avalanche gains greater than $5 \times 10^{5}$, and high responsivities. Similarly, the IR spectral band is useful for measuring ocean temperatures, atmospheric aerosols, forest fires, etc. We are also developing room temperature operating graphene-enhanced $\mathrm{PbSe}$ midwave infrared (MWIR) detectors and focal plane arrays (FPAs). These compact and low-cost MWIR sensors can benefit various NASA remote sensing applications. Here we present recent results from these high performance UV- and IR-band detector and FPA technologies.
\end{abstract}

\section{GAN/ALGAN UV AVALANCHE PHOTODIODE ARRAY DEVELOPMENT}

Detection of shorter UV wavelengths allows for increased spatial resolution, smaller pixels, and larger formats. UV-APD devices have been developed in collaboration with Georgia Tech that are based on a high quality MOCVD technique and growth on latticematched $\mathrm{GaN}$ substrates to demonstrate high performance. Figure 1 shows a schematic crosssectional view of the epitaxial layers and device structure for the $\mathrm{Al}_{0.05} \mathrm{Ga}_{0.95} \mathrm{~N}$ UV-APDs, with the contact pads and circular mesa area of the photodiode shown in the inset.

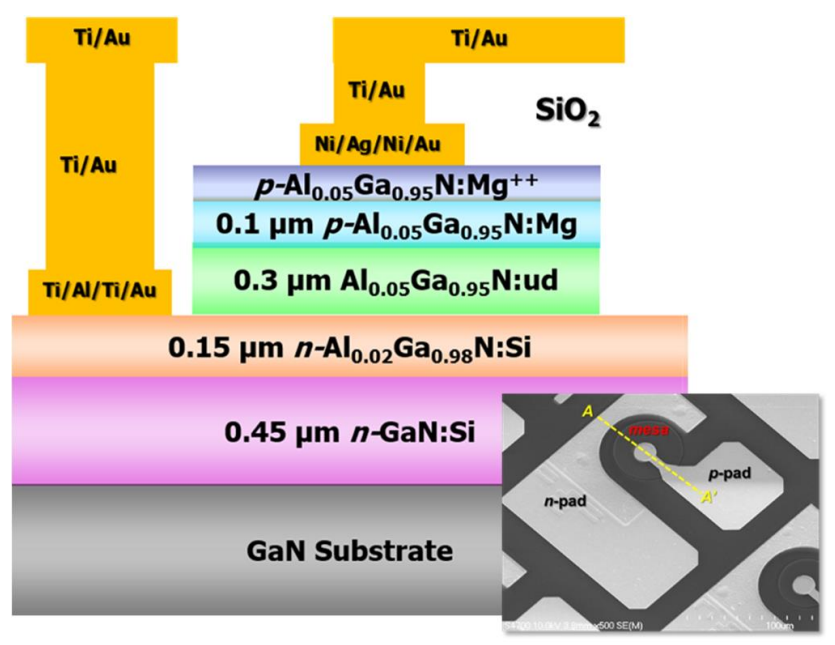

Figure 1. Device structure cross-section of GaN $p-i-n$ APD on bulk GaN substrate; inset shows top-view SEM image of device [1].

Figure 2 presents the I-V characteristics and avalanche gain for a $40 \mu \mathrm{m}$ diameter $\mathrm{Al}_{0.05} \mathrm{Ga}_{0.95} \mathrm{~N} \mathrm{UV}$ APD. Under dark conditions, the device demonstrated low dark current densities under $10 \mu \mathrm{A}$ (corresponding to dark current of $\sim 10^{-10} \mathrm{~A}$ ) up to a reverse voltage of around $60 \mathrm{~V}$ [2]. We believe that low defect density native substrates and high-quality MOCVD epitaxial growth technologies are key to the successful implementation of robust high gain, large-area UV detectors. 


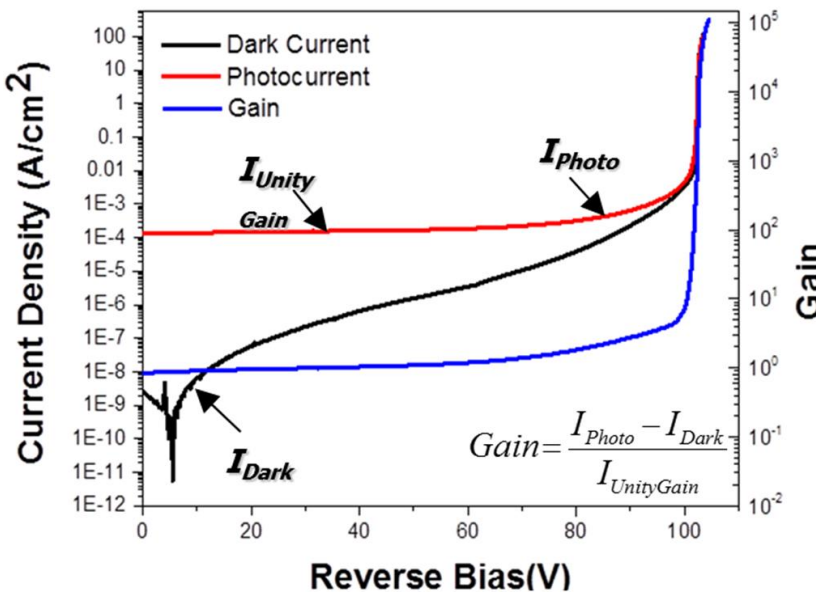

Figure 2. Current density and without UV illumination and gain plotted vs. reverse bias for $\mathrm{Al}_{0.05} \mathrm{Ga}_{0.95} \mathrm{~N}$ UVAPD with mesa diameter of $40 \mu \mathrm{m}$.

The detection wavelength of interest in the UV band is $355 \mathrm{~nm}$. UV detection devices operating at this YAG laser wavelength are very achievable with the $\mathrm{AlGaN}$ material system. We are currently working on optimizing the GaN/AlGaN material growth for $355 \mathrm{~nm}$ peak detector performance.

Figure 3 shows the wavelength-dependent responsivity of a $70 \mu \mathrm{m}$ diameter AlGaN UV-APD as a function reverse bias [3]. This device demonstrated a peak responsivity of $43 \mathrm{~mA} / \mathrm{W}$ at $\sim 355 \mathrm{~nm}$ under zero bias, corresponding to $94 \%$ quantum efficiency.

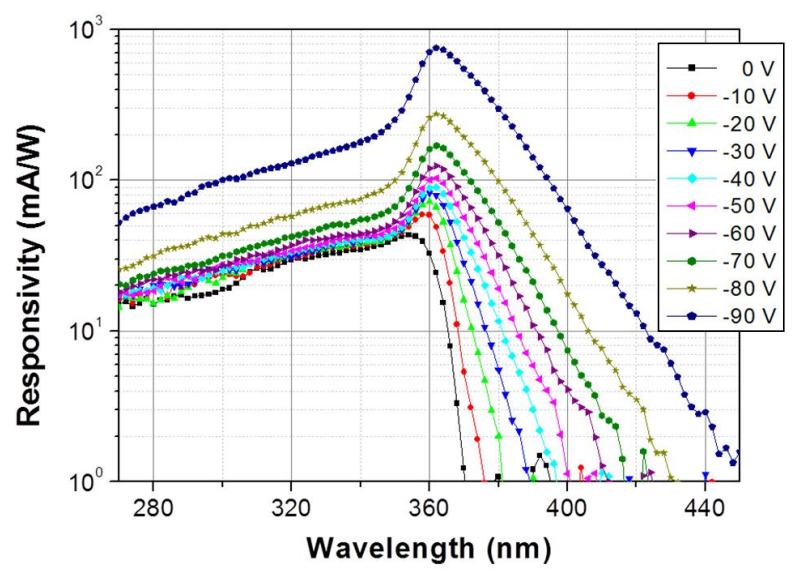

Figure 3. Reverse-biased-dependent spectral response of photocurrent measured at room temperature for 70 $\mu \mathrm{m}$ diameter $\mathrm{Al}_{0.05} \mathrm{Ga}_{0.95} \mathrm{~N}$ UV-APD, showing peak responsivity at $\sim 355 \mathrm{~nm}$ under zero bias [3].
Low defect density and low leakage current UV-APD devices have been fabricated by MOCVD. High avalanche gains have been demonstrated, with stable and repeatable operation. These GaN/AlGaN UVAPDs have the potential to advance various NASA applications such as satellite lidar.

\section{GRAPHENE ENHANCED MWIR PHOTODETECTOR AND FPA DEVELOPMENT}

Infrared detector and focal plane array (FPA) technologies have proven to be at the heart of many remote sensing instruments for numerous NASA missions. For Mid Wave Infrared wavelength (MWIR) detection it is very desirable to develop infrared detector technologies that operate at or near room temperature to minimize the cooling requirements.

The 2-5 $\mu \mathrm{m}$ MWIR spectral band is useful for measuring sea surface temperature, cloud properties, volcanic activities and forest fires, among other applications. Using low size, weight, power and cost MWIR sensors on smaller platforms in low orbit can enable improved measurements of thermal dynamics with high spatial resolution [4]. Polycrystalline materials such as PbSe have shown promise for MWIR detection for applications including long-range imaging, detection of molecular and biological agents, and early threat detection [5].

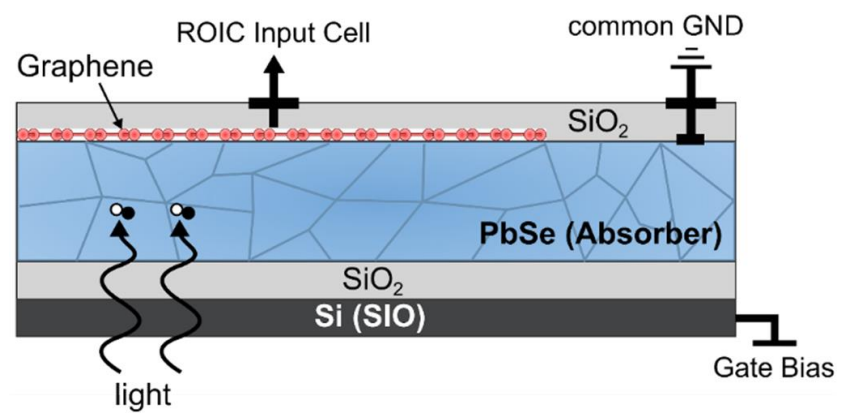

Figure 4. PbSe-graphene heterostructure IR photodetector structure.

We are developing a MWIR detector technology with graphene-PbSe photodetectors that combines the best features of both materials to provide higher performance compared to PbSe-only devices. The 
graphene-PbSe detector structure, shown schematically in Figure 4, is composed of three principle layers: $\mathrm{Si} / \mathrm{SiO}_{2}$ gate, $\mathrm{PbSe}$ absorber, and the graphene channel.

In this device, the electrically tunable properties of the graphene (such as doping level and carrier concentration) can be adjusted to modulate its carrier transport properties. Through applying a gate voltage bias, the intrinsic interfacial barriers between the PbSebased absorber and the graphene act as a tunable rectifier that reduces recombination of photogenerated carriers in the detector. The graphene also operates as high mobility channel that whisks away carriers before they can recombine, further enhancing the MWIR detection performance.

The goal in modeling these devices is to generate accurate electrical behavior and performance metrics and device design and operating specifications, allowing for design optimization considering constraints. In Figure 5, the simulated dark current and photocurrent for different drain-to-source biases is plotted. It is seen that shining light onto the detector significantly increases the drain current, which is crucial for photosensitivity.

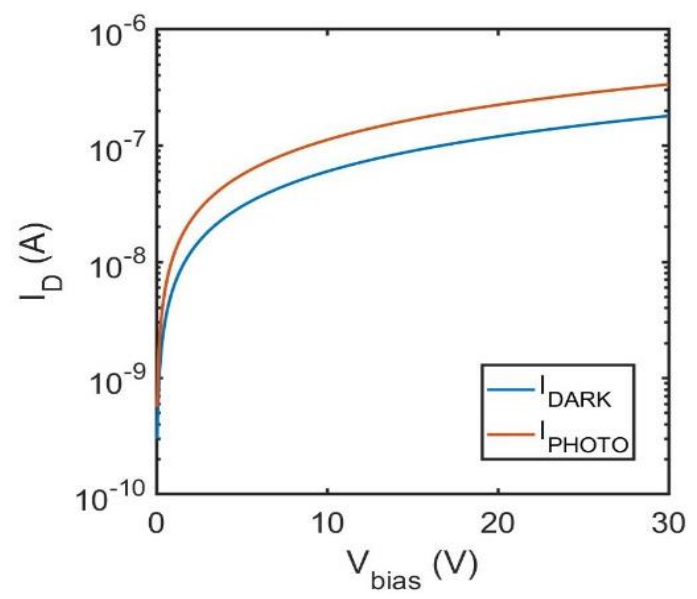

Figure 5. Simulated dark current and photocurrent of detector with $\mathrm{PbSe}$ absorber material.

The behavior of the graphene-PbSe MWIR photodetectors is largely dependent on the graphene doping condition and buildup of electrical potential due to incident photons. Figure 6(a) shows the shift in the I-V characteristics after IR radiation is incident on the sample, enabling sensing to occur. Figure 6(b) plots the simulated drain current vs. time for undoped graphene with and without light and with positive and negative applied gate voltages. These results show the significant effect the gate voltage has on the current in the MWIR detectors under both dark and illuminated conditions.
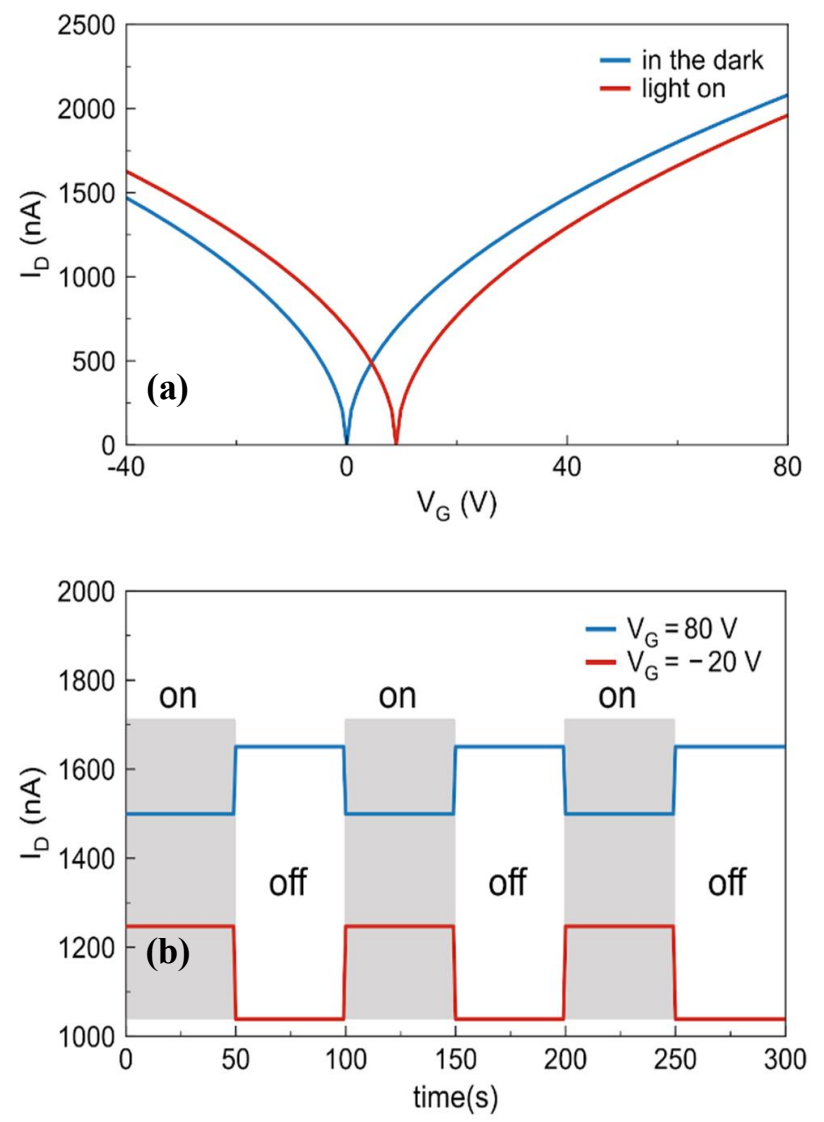

Figure 6. Simulation of sensing using graphene: (a) Drain current as function of gate voltage under light and dark conditions; (b) current plotted over time with alternating on-off IR illumination.

These exciting AlGaN UV-APD and PbSe-graphenebased photodetector and FPA technologies are being developed for high performance UV and IR sensing to support and further advance a variety of NASA Earth Science applications.

\section{REFERENCES}

[1] A. K. Sood, J. W. Zeller, Y. R. Puri, R. D. Dupuis, T. Detchprohm, M.-H. Ji, S. C. Shen, S. Babu, N. K. Dhar, and P. Wijewarnasuriya, "Development of high gain GaN/AlGaN avalanche photodiode arrays for UV detection and imaging 
applications," Int. J. Engr. Res. Tech., vol. 10, no. 2, pp. 129$150,2017$.

[2] J. Kim, M.-H. Ji, T. Detchprohm, J.-H. Ryou, R. D. Dupuis, A. K. Sood, and N. K. Dhar, " $\mathrm{Al}_{x} \mathrm{Ga}_{1-x} \mathrm{~N}$ ultraviolet avalanche photodiodes with avalanche gain greater than $10^{5}$," IEEE Photon. Tech. Lett., vol. 27, no. 6, pp. 642-645, 2015.

[3] A. K. Sood, J. W. Zeller, P. Ghuman, S Babu, R. D. Dupuis, and H. Efstathiadis, "Development of high performance ultraviolet and near-infrared detector technologies," Proc. SPIE, vol. 10766, p. 1076609, Sept. 2018.

[4] M. H, Jang, S. S. Yoo, N. K. Dhar, and M. C. Gupta, "High photo response in PbSe IR detectors by laser annealing." II-VI Workshop Proc., pp. 57-58, Nov. 2018.

[5] J. Grayer and S. S. Yoo, "Investigation of nano plasmonic antenna for increased performance of high temperature operation PbSe photoconductors," Proc. SPIE, vol. 10722, p. 107222 X, Sept. 2018. 